\title{
The New Pretender: A Large UK Case Series Of Retinal Injuries in Children Secondary to Hand-Held Lasers
}

Naz Raoof ${ }^{1}$, Patrick Bradley², Maria Theodorou ${ }^{2}$, Anthony T. Moore ${ }^{2,3}$, Michel Michaelides ${ }^{2,4^{*}}$

1. Department of Ophthalmology, Faculty of Medical and Health Sciences, The University of Auckland, Bldg 504, Level 4, Cnr of Boyle Cr \& Glasgow Tce, Park Road, Grafton, Auckland, New Zealand

2. Moorfields Eye Hospital NHS Foundation Trust, 162 City Road, London, UK EC1V 2PD

3. Ophthalmology Department, UCSF School of Medicine, Koret Vision Centre, 10 Koret Way, San Francisco. CA 94133

4. University College London Institute of Ophthalmology, 11-43 Bath Street, London EC1V 9EL, UK

Short title: UK series of retinal laser injuries in children

* Corresponding author:

Professor Michel Michaelides, Moorfields Eye Hospital NHS Foundation Trust, 162 City Road, London, EC1V 2PD

Telephone: 00442076086864

Fax: 00442076086903

Email: michel.michaelides@ucl.ac.uk 


\section{Introduction}

There are increasing reports of retinal injuries secondary to hand-held lasers, with cases found across the world ${ }^{1-6}$. These hand-held lasers often resemble laser-pointers, although they are commonly marketed as toys ${ }^{1}$. While certain safeguards exist to protect consumers (i.e. FDA safety notification in the US and BS EN 60825-1:2014 in the UK), these are difficult to police as devices are often purchased outside the country of use, and often from an on-line retailer ${ }^{1}$. Moreover, labeling of laser devices may be misleading.

Most descriptions of retinal injuries secondary to hand-held laser devices come from single case reports, or relatively small case series, so the retinal damage is not well characterized. Additionally, such laser devices are attractive to children, who may give a vague history following an injury or deliberately withhold information for fear of being punished. The diagnosis may be missed unless specific questions about access to such lasers are asked.

In the acute phase, several reports describe a vitelliform-like maculopathy, resolving to leave a laser 'scar'1,3. Alsulaiman et al. describe 17 patients who sustained macular holes following exposure to high power blue hand-held lasers ${ }^{7}$, while Yiu et al describe pre-retinal macular haemorrhages ${ }^{8}$. The visual impact of these retinal laser injuries is variable, with some reports describing severe, long-term effects on visual function ${ }^{9}$, while other injuries resolve to near normal levels of visual acuity, although more subtle visual deficits may remain ${ }^{10}$. The aim of this study was to characterize a relatively large series of children seen at a single center, who sustained retinal injuries secondary to hand-held laser devices. We describe common features in the presentation of patients, the clinical spectrum of injury, as well as the appearance of injuries on spectraldomain Optical Coherence Tomography (OCT) and the visual outcome. Our findings should aid early diagnosis, improve patient counseling and advice on prognosis, and provide data that regulatory authorities might use to better protect children from retinal damage and potential long-term visual impairment. 


\section{Methods}

Approval to undertake this observational case series was obtained by our institutional review board at Moorfields Eye Hospital NHS Foundation Trust. In line with our institutional policy, consent was not specifically sought, due to the retrospective nature of the study and that all data was anonymised. Patients with retinal injuries secondary to hand-held laser devices were identified by searching our electronic patient record system at Moorfields Eye Hospital (London, UK) between $1^{\text {st }}$ January 2011 to $30^{\text {th }}$ June 2015. A retrospective casenotes review was undertaken for patients identified, as well as a review of retinal imaging. Patients underwent pupillary dilation prior to OCT and Fundus Auto-Fluorescence (FAF) imaging, using a Spectralis ${ }^{\mathrm{TM}} \mathrm{FAF}+$ OCT device (Heidelberg Engineering(C), Heidelberg, Germany). Statistical analysis was undertaken using an Excel Spreadsheet. 


\section{Results}

We identified 16 children, (24 affected eyes), with retinal injuries secondary to laser devices. Of these, $12(75 \%)$ were male and 4 were female. The mean age was 12.7 years (range 9-16 years). There were 8 (50\%) bilateral cases. All affected children were from the United Kingdom, although 5 (31\%) of the cases were injuries sustained abroad; in 2 cases the exposure occurred in China, 1 case in Greece and 1 in France, with the 5th case documented only as 'abroad'. Clinical findings are summarized in Table 1.

Features in the clinical history

A delay in the diagnosis of a laser injury was a common feature among our patients. Five (31\%) children were referred for a specialist opinion with suspected retinal dystrophies. Best disease was thought to be the likely diagnosis in 3 cases and Stargardt disease suspected in one child. One child had been seen in an ocular genetics clinic, but had not received any formal diagnosis. Furthermore, children frequently denied exposing themselves to a laser pointer or having a laser shone into their eyes when asked; with only $8(50 \%)$ children admitting this (in one case this admission occurred 7 months after first consultation). A further 7 children admitted that they had played with a laser toy, or their parents admitted that they had kept a laser toy in the house. One child, with a retinal appearance that strongly suggested a laser injury (figure 1), to date has denied laser exposure. In 5 (31\%) cases, the causative laser was a green laser; in one other known case the causative laser was red.

The most commonly encountered symptom was a central scotoma (11 children, $69 \%$ ), followed by blurred vision (3 children, 19\%). Two children (12\%) were asymptomatic and referred because their optometrist noted macular changes. The mean follow up period was 5.4 months (range 1 to 23 months).

\section{Examination and Imaging findings}

The mean logMAR visual acuity at presentation was $0.30(20 / 40)$ (range -0.20 $(20 / 12.5)$ to $1.6(20 / 800))$. A wide spectrum of retinal appearances was encountered. OCT imaging was available for 15 (94\%) patients; one child was seen in our emergency department with a retinal laser injury that had been diagnosed in another department and no OCT images were taken at this visit. Thus in two eyes OCT classification of the injury was not possible.

Injuries were classified as mild if they were focal in nature, with small discrete outer retinal and retinal pigment epithelium (RPE) disturbance at the macula; a typical macular appearance is shown in figure 2. OCT imaging in these injuries confirmed the presence of focal retinal disruption confined to the photoreceptor and ellipsoid layers. Eleven (69\%) children, corresponding to 15 eyes, had 'mild' injuries. The mean visual acuity in this subset was $0.10(20 / 25)$ (range -0.20 $(20 / 12.5)$ to $0.60(20 / 80))$. One patient in this group with a unilateral injury had a 'microhole' appearance with focal loss of subfoveal photoreceptors; the visual acuity was $0.20(20 / 32)$ in this child. The majority of injuries in this category were unilateral $(8,80 \%)$. One child with a 'mild' retinal injury in one eye had a 'severe' retinal injury in the other. 
Injuries were considered 'moderate' if retinal disruption was confined to the outer retinal layer but diffuse rather than focal in nature (figure 3). Three eyes of two children had a 'moderate' severity injury. One child had bilateral 'moderate' severity injury. This child was resident in China at the time of injury, but returned to the United Kingdom for further management. When he was seen in our unit, his visual acuities were $0.48(20 / 60)$ and $0.96(20 / 180)$ in the right and left eyes respectively. The other child had a 'moderate' severity injury in the left eye with a logMAR visual acuity of 1.14 (20/320) and a 'severe' injury in the other eye (see below).

Three patients (4 eyes) had 'severe' injuries, with subfoveal loss of outer retinal architecture and overlying hyper-reflective material in inner retinal layers (figure 4). These injuries were associated with worse vision, the mean logMAR visual acuity being $1.1(20 / 250)$ (range $0.80(20 / 125)$ to $1.6(20 / 800))$. One patient had bilateral 'severe' injuries, one child had a 'severe' injury in one eye and a 'moderate' injury in the other (figure $1 \mathrm{a}$. and b.) and one child had a left 'severe' injury with a visual acuity of $1.6(20 / 800)$ and a mild injury in the other eye with a visual acuity of $0.0(20 / 20)$.

Over the follow-up period, we observed that patients with good visual acuity at presentation and 'mild' injuries retained good vision. Patients with more extensive injuries also showed a tendency to stay stable, although we did not observe visual improvement over our follow-up period. One child with bilateral 'moderate' severity injuries showed evidence of worsening vision, as his logMAR visual acuity at the time of referral in China was $0.40(20 / 50)$ and $0.20(20 / 32)$ in the right and left eyes respectively, but had deteriorated to $0.48(20 / 60)$ and $0.96(20 / 180)$ two months later, when seen at our unit. The mean logMAR visual acuity at final follow-up was $0.34(20 / 40)$ (range $-0.2(20 / 12.5)$ to 1.6 $(20 / 800))$. 


\section{Discussion}

We report a large series of retinal injuries in children secondary to recreational laser devices seen at a single unit. We propose a system for classifying such retinal injuries, based on OCT findings. Our study also provides valuable information to clinicians, which may aid the early diagnosis of such injuries and the provision of advice on prognosis.

Our study shows that there is frequently a delay in making the diagnosis of a retinal laser injury. One contributory factor is that such injuries often affect children who frequently deny exposure to laser toys, presumably due to a fear of punishment. 'Milder' injuries, which comprised over half of injuries seen, tend to be unilateral with good vision in the affected eye as well as a normal unaffected eye. The fact that many affected children have relatively good vision could also lead to a delay in voicing their symptoms and hence a delay in seeking medical advice at a point when the 'vitelliform-like' appearance of an acute laser injury ${ }^{1}$ may aid a diagnosis compared with the more non-specific atrophic changes in an established retinal injury. The lack of awareness of such injuries amongst Ophthalmologists is likely to contribute towards a delay in making this diagnosis, as evidenced by the fact that almost a third of children in this study were referred as suspected retinal dystrophies. It is crucial that clinicians are aware of the clinical features of laser injury so that a specific targeted history can be taken and unnecessary investigations can be avoided.

The differential diagnosis for laser injuries includes Best disease, Stargardt disease, cone-rod dystrophies, solar retinopathy and infective causes including toxoplasma chorioretinitis. In our series, Best disease was thought to be the diagnosis in 3 children. In the acute stages of laser injuries, there is often a 'vitelliform-like' change at the macula. Unlike Best disease, there is no subretinal deposit, although outer retinal disruption is seen during the acute phase ${ }^{1}$. The 'vitelliform-like' changes also resolve in laser injuries over as short a period as 3 days, leaving areas of RPE disturbance ${ }^{1}$. One case in our series with bilateral moderate severity laser burns was diagnosed as having Stargardt disease when he was seen in China. We are unable to comment about the clinical picture at the time of his presentation. In the very early stages of Stargardt disease, where only macular atrophy may be present, the appearance may have some similarities to a laser injury. However, the discrete focal outer retinal loss often seen in mild-moderate laser injury, and the presence/development of yellowwhite retinal flecks and often a bulls-eye maculopathy-like appearance in Stargardt disease, aids the diagnosis. It is plausible that a laser injury could be misdiagnosed as a cone-rod dystrophy, although the absence of peripheral retinal disturbance and generalized retinal dysfunction on electrophysiological testing will differentiate the two conditions. Similarly, there are no reports of intraocular inflammation in children with laser injuries, hence inflammatory pathologies can be discounted in a straightforward manner. Solar retinopathy may be difficult to distinguish clinically from a laser injury, as a yellowish macular lesion may be seen acutely, followed by similar outer retinal changes in milder solar injuries, with inner retinal changes in more extensive injuries ${ }^{11}$. This highlights the importance of the history in children with laser injuries, and clinician awareness. 
We have classified injuries into 'mild', 'moderate' and 'severe' categories based on OCT findings. The likely mechanism for the retinal injuries seen is in keeping with the mechanism via which panretinal photocoagulation takes effect; laser energy is absorbed by the RPE layer, with the subsequent heating up of this layer resulting in destruction of the overlying retinal layers. We postulate that the severity of the laser burn is determined by the amount of laser energy absorbed by the RPE. Likely factors contributing to the severity of a laser injury include the different wavelengths and powers of causative devices and different lengths of exposure. 'Mild' injuries comprise the majority of injuries seen in our series and tended to correlate with better visual acuity and carry a better prognosis. This corresponds with a survey of Euretina members on this topic, which reported that $65 \%$ of injuries had no effect or mild effect on vision (6/9-6/12) (communication from F.Quhill, survey presented at Euretina 2015).

Only $12.5 \%$ of injuries in our series were 'moderate' and $17 \%$ were 'severe'. This makes it harder to draw firm conclusions about the prognosis of such injuries, or whether indeed these two categories behave in a similar clinical manner despite having different appearances on OCT. Our study did not show an improvement in visual function of children with more extensive injuries, but our follow-up period was relatively short at a mean of 5.4 months. Lee et al. report 2 children with poor visual acuity and more extensive retinal injury ${ }^{3}$. Both children showed some improvement in their visual acuity over a year; one child improved from counting fingers to 20/100 and 20/70 in the right and left eyes respectively and the other child from counting fingers to 20/100 in the affected eye. Similarly, Lally et al. report a 9-year old child with 'severe' laser induced injuries whose visual acuity improved slightly from counting fingers in each eye to 20/200 over six months ${ }^{9}$. In an early review of laser-induced retinal injuries in 2012, Turkana et al. report that the visual acuity at final follow-up in 10 individuals ranged from $20 / 20$ to $20 / 60$, although the visual acuities at presentation were also better, with only one patient having counting fingers vision ${ }^{6}$. OCT findings are not available from this study to assess the structural severity of these injuries. A recent study by Quhill's group in the UK reported that 2 children with OCT evidence of a more extensive retinal injury demonstrated an improvement in visual acuity from around $6 / 15$ to $6 / 9$ over a 12 -month period ${ }^{10}$. Longer followup of such more extensive injuries is likely to prove useful in counseling affected patients in the future.

Even within a particular category of retinal injury, the visual acuity remains variable. Within the 'mild' injuries, there were children with a logMAR vision of $-0.20(20 / 12.5)$ to $0.60(20 / 80)$. The child with a visual acuity of $0.60(20 / 80)$ had a single area of focal outer segment and ellipsoid layer disruption, although this was at a subfoveal location. At 6 months follow-up, however, this child's visual acuity had improved to $0.10(20 / 25)$. Similarly, it remains unclear why the child with bilateral 'moderate' retinal injuries should have worsening visual acuity from $0.40(20 / 50)$ and $0.20(20 / 32)$ in the right and left eyes respectively to $0.48(20 / 60)$ and $0.96(20 / 180)$ two months later. Unfortunately the OCTs and color fundus images at the first time point were not available for review. We speculate that the expansion of chorio-retinal scarring in this child may have contributed to the worsening vision. 
We acknowledge that there are limitations associated with our study. Although we report a large series of such injuries in children encountered at a single center, we acknowledge that 24 eyes of 16 patients is still a relatively small population. We also appreciate that a longer follow-up interval will prove useful in fully establishing the natural history of these injuries. Further measure of visual function may also be very useful, for instance contrast sensitivity and microperimetry ${ }^{10}$, in establishing long-term deficits.

Retinal laser injuries, while potentially devastating, are entirely avoidable. A key consideration is raising awareness of this entity amongst parents, teachers and children. In the United States, the Food and Drug Administration have released videos such as the "Laser Pointer Safety Video", available to the public online, with recommendations that include not shining lasers directly into the eyes, refraining from giving children laser pointers to play with and checking that any laser is less than $5 \mathrm{~mW}$ in power. Additionally, we would echo advice against buying any laser device from an on-line marketplace or from a street vendor, where the power of a laser device may be mislabeled ${ }^{10}$. We would also support more public health campaigns directly aimed at schoolchildren, warning against the risk posed by laser devices. Our recommendation is that any Ophthalmologist who encounters a patient with a laser injury should report this to the relevant authority in their country, as better reporting of such injuries is likely to encourage action on the part of lawmakers. The FDA, for example, encourages any suspected laser injury to be reported through MedWatch, even if sustained from a non-medical laser.

In conclusion, we present a large series of children seen at a single unit with macular injuries secondary to recreational laser devices. There is frequently a delay in clinicians making this diagnosis, which leads us to believe such injuries are under-diagnosed. Injuries can range in severity, from relatively mild injuries that are likely to be associated with a better prognosis, to more extensive injury that may leave children with life-changing visual deficits. It is important that such data are in the public domain so that regulatory authorities recognize the importance of laser retinopathy as an avoidable cause of childhood visual impairment and take steps to minimize the incidence and impact of laser injuries. 


\section{Acknowledgements}

A. Funding support: The work was supported by grants from the National Institute for Health Research Biomedical Research Centre at Moorfields Eye Hospital National Health Service Foundation Trust and UCL Institute of Ophthalmology, Moorfields Eye Hospital Special Trustees (ST 1310 F), and Moorfields Eye Charity (MEC 1310 A). Prof. Michaelides is a recipient of a Foundation Fighting Blindness Career Development Award (CD-CL-0711-0518UCL).

B. Financial disclosures: No financial disclosures.

C. Other Acknowledgements: None. 


\section{References:}

1. Raoof N, Chan TK, Rogers NK et al. Toy laser macular burns in children. Eye (Lond). 2014;28(2):231-234

2. Alsulaiman SM, Alrushood AA, Almasaud J et al. High-power handheld blue laser-induced maculopathy: the results of the King Khaled Eye Specialist Hospital Collaborative Retina Study Group. Ophthalmology. 2014;121(2):566-572

3. Lee GD, Baumal CR, Lally D, Pitcher JD, Vander J, Duker JS. Retinal injury after inadvertent handheld laser exposure. Retina. 2014;34(12):23882396

4. Weng CY, Baumal CR, Albini TA, Berrocal AM. Self-induced laser maculopathy in an adolescent boy utilizing a mirror. Ophthalmic Surg Lasers Imaging Retina. 2015;46(4):485-488

5. Dhoot DS, Xu D, Srivastava S. High-powered laser pointer injury resulting in macular hole formation. J Pediatr. 2014;164(3):668

6. Turaka K, Bryan JS, Gordon AJ, Reddy R, Kwong HM Jr, Sell CH. Laser pointer induced macular damage: case report and mini review. Int Ophthalmol. 2012;32(3):293-297

7. Alsulaiman SM, Alrushood AA, Almasaud J et al. Full-Thickness Macular Hole Secondary to High-Power Handheld Blue Laser: Natural History and Management Outcomes. Am J Ophthalmol 2015;160(1):107-113

8. Yiu G, Itty S, Toth CA. Ocular safety of recreational lasers. JAMA Ophthalmol. 2014;132(3):245-246

9. Lally DR, Duker JS. Foveal injury from a red laser pointer. JAMA Ophthalmol. 2014;132(3):297

10. Raoof N, O'Hagan J, Pawlowska N, Quhill F. 'Toy' laser macular burns in children: 12-month update. Eye (Lond) 2016;30(3):492-496

11. Gregory-Roberts E, Chen Y, Harper CA et al. Solar retinopathy in children. J AAPOS 2015;19(4):349-51 


\section{Figure Legends}

Figure 1, top

Colour fundus photographs of a 15-year-old girl with bilateral decreased visual acuity (logMAR $1.3(20 / 400)$ and $1.14(20 / 320)$ right and left eyes respectively). Extensively investigated for inflammatory, infectious and metabolic causes elsewhere, which all proved negative. Electrophysiological testing revealed a normal ERG and Pattern ERG, with abnormal multifocal ERG only in the region of the lesion. Fluorescein angiography and indocyanine green angiography yielded no further information.

Figure 1, bottom

Right eye spectral domain OCT image of the patient, showing profound right subfoveal and parafoveal disruption extending from the photoreceptor outer segment layer to the outer nuclear layer, with inner retinal hyperreflective material.

Figure 2, top

Right eye color fundus photograph to show focal macular laser burns, seen as discrete areas of RPE change; LogMAR visual acuity was -0.1 (20/16).

Figure 2, bottom

Right eye spectral domain OCT image of the patient, showing focal retinal disruption confined to the photoreceptor and ellipsoid layers.

Figure 3, top

A left eye color fundus photograph of a 13-year-old boy, showing an area of outer retinal atrophy centered over the macula.

Figure 3, bottom

A left eye OCT image of the patient, showing diffuse outer segment and ellipsoid layer disruption with outer retinal deposition of highly reflective material.

Figure 4, top

A right eye colour fundus photograph of a 12-year-old boy with bilateral decreased visual acuity (logMAR $0.8(20 / 125)$ both eyes) showing foveal and parafoveal atrophic lesions in keeping with laser burns; left eye appearance similar.

Figure $4 \mathrm{~b}$, bottom

A right eye spectral domain OCT showing subfoveal loss of outer retinal architecture and overlying hyper-reflective material in inner retinal layers. 